\title{
Clinical profile of patients with 46 XY disorders of sex development: a single centered experience
}

\author{
Darvin V. Das* \\ Department of Endocrinology, Government Medical College, Thiruvananthapuram, Kerala, India \\ Received: 03 March 2021 \\ Accepted: 06 April 2021 \\ *Correspondence: \\ Dr. Darvin V. Das, \\ E-mail: drdarvindas@gmail.com \\ Copyright: (C) the author(s), publisher and licensee Medip Academy. This is an open-access article distributed under \\ the terms of the Creative Commons Attribution Non-Commercial License, which permits unrestricted non-commercial \\ use, distribution, and reproduction in any medium, provided the original work is properly cited.
}

\begin{abstract}
Background: XY disorders of sex development are a complex entity that needs medical attention from childhood to adolescence and throughout life. The aim of the study was to analyze retrospectively the medical records of subjects with $46 \mathrm{XY}$ disorders of sex development (DSD) and characterize their clinical profile and management course in a tertiary care centre.

Methods: 32 subjects with 46 XY DSD attending Endocrinology OPD / Gynecology between 2010 to 2020 were enrolled in the study. Data collected includes age at presentation, symptoms, sex of rearing, phenotype, external masculinisation score (EMS), karyotyping, gonadal features like location and histopathology, psychosexual domain and their management and follow up. Statistical Analysis: The mean and standard deviation was calculated for normally distributed data.

Results: The mean (SD) age of all study subjects $(n=32)$ were $15.5 \pm 5.32$ years. 46 XY DSD included cases of complete gonadal dysgenesis $(n=6)$, mixed gonadal dysgenesis $(n=6)$, complete androgen biosynthetic defects $(n=9)$, partial gonadal dysgenesis $(n=2), 5$-alpha-reductase type 2 deficiency $(n=8)$ and 17 betahydroxysteroid deficiency $(\mathrm{n}=1)$. The most common clinical presentation was for primary amenorrhea followed by genital ambiguity and virilisation in females. Resection of testis at the earliest or a biopsy of the testis if resection is postponed in a female sex assigned 46 XY DSD is favored. Male sex assigned 46 XY DSD needs corrective surgeries and orchidopexy for undescented viable testis with periodic follow up for testicular malignancy.

Conclusions: 46 XY DSD's may shares similarities in their clinical presentation, though age of presentation may be in a wide range. Proper gender assignment, gonadectomy, reconstructive surgeries, hormone replacement and time to time follow up for testicular malignancy in cases where the testes are preserved is the ideal management.
\end{abstract}

Keywords: Clinical profile, Hormone replacement therapy, Reconstructive surgeries, XY disorders of sex development

\section{INTRODUCTION}

$46 \mathrm{XY}$ disorder of sex development (DSD) consists of spectrum disorders where males are undervirilised due to defective gonadal development, androgen biosynthesis or its androgen action. ${ }^{1}$ At birth, they may present with ambiguous genitals, salt wasting crisis, undescented testis or absent testis. A few cases may remain undetected until they reach puberty. At puberty they present as primary amenorrhea in girls or undervirilisation in boys. Individuals with $46 \mathrm{XY}$ DSD presents to the clinician at different age and the management of DSD is complex and needs a multi-disciplinary approach. In this study, we aim to analyze retrospectively the medical records of subjects with $46 \mathrm{XY}$ DSD and characterize clinical features and study their management issues from a tertiary care center. 


\section{METHODS}

The study was conducted in the department of Endocrinology in our tertiary care hospital between 2010 to 2020 after receiving approval from the ethics committee of the institute HEC.No.05/18/2020/MCT. All subjects who had been seeking treatment for disorders of sex development were collected from records library. Individuals who had a karyotype $46 \mathrm{XY}$ disorder of sex development (DSD) were enrolled for the study. Our study included 32 subjects with XY DSD. XY DSD included complete gonadal dysgenesis (CDG), partial gonadal dysgenesis (PGD), 45,X/46 XY mosaicism or mixed gonadal dysgenesis (MGD), androgen biosynthetic defects [(5-alpha reductase deficiency and 17 betahydroxysteroid deficiency (17 $\beta$-HSD3)] and complete androgen insensitivity syndrome (CAIS). Clinical characteristics collected included age at presentation, clinical symptoms, sex of rearing, phenotype, external masculinisation score (EMS), karyotyping, MRI- abdomen and pelvis, hormonal profile, location of gonads and the type of internal ducts based on the laparoscopic or laparotomy findings, histopathology of the gonads, psychosexual domain and the management mode. 46 XY CGD was diagnosed in an individual with female phenotype, complete absence of testicular tissue (streak gonad on histology) with the presence of normal or rudimentary Mullerian structures. $46 \mathrm{XY}$ PGD was diagnosed as dysgenetic testis (on histology) with genital ambiguity with varying degree of wolffian or / and Mullerian ducts. MGD was diagnosed based on genital phenotype, gonadal phenotype and mosaic 45, X/46, XY karyotype. Genital phenotype included from female external genitalia, mild clitoromegaly or ambiguous genitalia. Gonadal phenotype included streak gonads in one side and a dysgenetic testis to normal testis in other side. Androgen biosynthetic defects (ABD) were diagnosed based on genital phenotype, gonadal position and ratio of principle hormone to its precursor. In hCG stimulation test baseline blood samples were collected in fasting for total testosterone. hCG was administered deep intramuscular for three consecutive days in a dose based on the age of the patient (age $<1$ year 500 IU per day hCG, age 1-10 years 1000 IU per day and age $>10$ years 1500 IU per day). Blood samples were collected 24 hours after the third dose of total testosterone $(\mathrm{T})$, dihydrotestosterone (DHT) and androsteinedione (A). Ratios of stimulating hormones T/DHT ratio $>20$ indicates steroid 5-alphareductase type 2 enzyme deficiency, while a T/A ratio $<0.8$ is suggestive of $17 \beta-H S D 3$ enzyme deficiency. ${ }^{2}$ Androgen receptor mutation analyses were not available in the center to diagnose androgen insensitivity. CAIS was diagnosed based on female phenotype with $46 \mathrm{XY}$ karyotyping with gonads being normal testis on histology and positive androgen insensitivity test (AIT). AIT is done with oral stanozolol $(0.2 \mathrm{mg} / \mathrm{kg} /$ day $)$ and nadir levels of sex hormone binding globulins at day 5 to 8 days from 93-97\% indicate CAIS and not an androgen biosynthetic defect. $^{2}$ No cases of $46 \mathrm{XY}$ DSD with CAH was available. EMS was devised to see the degree of under virilization among the DSD cases. ${ }^{3}$

\section{Statistical analysis}

The mean and standard deviation was calculated for normally distributed data. $\mathrm{P}$ value $<0.05$ was considered significant. Results were analysed with the SPSS software 16.

\section{RESULTS}

The mean (SD) age of all study subjects $(n=32)$ was 15.5 \pm 5.32 years (range 2- 28 years). There were six cases of CGD, six cases of MGD, nine cases of CAIS, two cases of PGD, eight cases of 5-alpha-reductase type 2 enzyme deficiencies and one case of 17ß-HSD3 enzyme deficiency. The most common clinical presentation was primary amenorrhea $(\mathrm{n}=12,38 \%)$ followed by genital ambiguity $(n=9,28 \%)$, clitoromegaly $(n=4,12, \%)$, primary infertility $(\mathrm{n}=6,19 \%)$ and gynecomastia $(\mathrm{n}=1$, $3 \%$ ). 22 children were assigned and raised as female whereas 10 children as males at birth Genital ambiguity was noticed among androgen biosynthetic defects and one case of MGD. Genital ambiguity was in the form of isolated penile hypospadia, bifid scrotum, chordee with hypospadia and perineoscrotal hypospadia with blind vaginal pouch. A child diagnosed as $17 \beta-$ HSD3 enzyme deficiency presented with gynecomastia at puberty. The mean height (SD) of study subjects was $148.1 \pm 8.2 \mathrm{~cm}$. Among all only one subject with MGD had short stature (height $128 \mathrm{~cm}, \mathrm{SDS}=-3.24$ ). The mean EMS score (SD) was $3.8 \pm 3.3$. All patients underwent a psychosexual assessment after diagnosis Table 2. Prior to gonadectomy all subjects underwent counselling to decide on the age preferences. Bilateral gonadectomy was done among CGD, MGD, CAIS and PGD who wished to continue as females. One case of MGD and all cases of ABD wished to continue as males. MGD who wished to continue as male (Number 12) underwent unilateral gonadectomy of intra-abdominal gonad in view of possible streak gonad non-functioning which has a high chance of malignancy and inguinal testis was biopsied and confirmed as testis and later underwent orchidopexy. Descended gonads are considered testis and hence in children with ABD scrotal gonads were considered testis even without histology. MGD male child and ABD children underwent corrective surgeries for hypospadia, correction of chordee and orchidopexy. The child with gynecomastia was started on with tamoxifen and found improvement. The surgical procedure was undertaken after multidisciplinary counselling. Children who completed 12 years among CGD, MGD, PGD and CAIS were started on estrogen therapy followed by cyclical estrogen and progesterone. Testosterone therapy was given to one child with 17 BHSD3 enzyme deficiency (Number 32) and MGD (Number 12). 5-alpha-reductase type 2 enzyme deficiency subjects were only given reconstruction surgeries and all had normal serum testosterone levels. 
Table 1: The characteristics of children with 46 XY DSD (n=32).

\begin{tabular}{|c|c|c|c|c|c|c|c|c|c|c|}
\hline 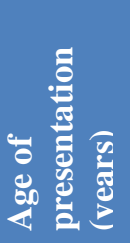 & 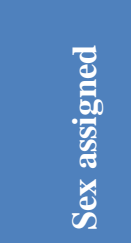 & 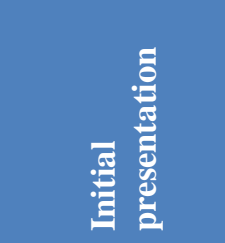 & 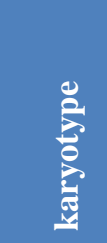 & 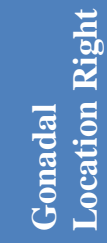 & 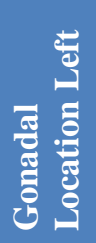 & $\sum_{x=1}^{\infty}$ & 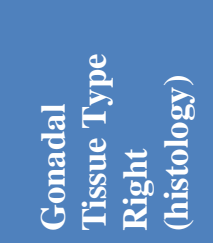 & 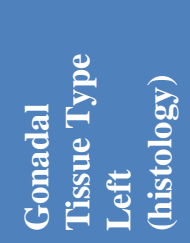 & 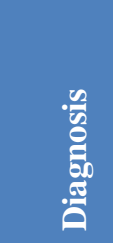 & 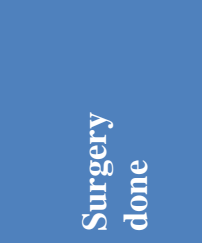 \\
\hline 14 & Female & $\begin{array}{l}\text { Primary } \\
\text { Ammenohrrea }\end{array}$ & $46 X Y$ & $\mathrm{AB}$ & $\mathrm{AB}$ & 1 & Streak & Streak & CGD & $\begin{array}{l}\text { Bilateral } \\
\text { gonadectomy }\end{array}$ \\
\hline 14 & Female & $\begin{array}{l}\text { Primary } \\
\text { Ammenohrrea }\end{array}$ & $46 X Y$ & $\mathrm{AB}$ & $\mathrm{AB}$ & 1 & Streak & Streak & CGD & $\begin{array}{l}\text { Bilateral } \\
\text { gonadectomy }\end{array}$ \\
\hline 14 & Female & $\begin{array}{l}\text { Primary } \\
\text { Ammenohrrea }\end{array}$ & $46 X Y$ & $\mathrm{AB}$ & $\mathrm{AB}$ & 1 & Streak & Streak & CGD & $\begin{array}{l}\text { Bilateral } \\
\text { gonadectomy }\end{array}$ \\
\hline 14 & Female & $\begin{array}{l}\text { Primary } \\
\text { Ammenohrrea }\end{array}$ & $46 X Y$ & $\mathrm{AB}$ & $\mathrm{AB}$ & 1 & Streak & Streak & CGD & $\begin{array}{l}\text { Bilateral } \\
\text { gonadectomy }\end{array}$ \\
\hline 16 & Female & $\begin{array}{l}\text { Primary } \\
\text { Ammenohrrea }\end{array}$ & $46 X Y$ & $\mathrm{AB}$ & $\mathrm{AB}$ & 1 & Streak & Streak & CGD & $\begin{array}{l}\text { Bilateral } \\
\text { gonadectomy }\end{array}$ \\
\hline 16 & Female & $\begin{array}{l}\text { Primary } \\
\text { Ammenohrrea }\end{array}$ & $46 X Y$ & $\mathrm{AB}$ & $\mathrm{AB}$ & 1 & Streak & Streak & CGD & $\begin{array}{l}\text { Bilateral } \\
\text { gonadectomy }\end{array}$ \\
\hline 4 & Female & Clitoromegaly & $\begin{array}{l}45 X / \\
46 X Y\end{array}$ & $\mathrm{AB}$ & $\mathrm{AB}$ & 4 & Streak & DT & MGD & $\begin{array}{l}\text { Bilateral } \\
\text { gonadectomy }\end{array}$ \\
\hline 8 & Female & Clitoromegaly & $\begin{array}{l}45 X / \\
46 X Y\end{array}$ & $\mathrm{AB}$ & $\mathrm{AB}$ & 4 & Streak & DT & MGD & $\begin{array}{l}\text { Bilateral } \\
\text { gonadectomy }\end{array}$ \\
\hline 10 & Female & Clitoromegaly & $\begin{array}{l}45 X / \\
46 X Y\end{array}$ & $\mathrm{AB}$ & $\mathrm{AB}$ & 4 & Streak & DT & MGD & $\begin{array}{l}\text { Bilateral } \\
\text { gonadectomy } \\
\text { and clitoral } \\
\text { recession }\end{array}$ \\
\hline 12 & Female & $\begin{array}{l}\text { Primary } \\
\text { Ammenohrrea }\end{array}$ & $\begin{array}{l}45 X / \\
46 X Y\end{array}$ & $\mathrm{AB}$ & $\mathrm{AB}$ & 4 & Streak & DT & MGD & $\begin{array}{l}\text { Bilateral } \\
\text { gonadectomy }\end{array}$ \\
\hline 14 & Female & Clitoromegaly & $\begin{array}{l}45 X / \\
46 X Y\end{array}$ & $\mathrm{AB}$ & $\mathrm{AB}$ & 1 & Streak & DT & MGD & $\begin{array}{l}\text { Bilateral } \\
\text { gonadectomy }\end{array}$ \\
\hline 16 & Male & $\begin{array}{l}\text { Ambigious } \\
\text { genitals }\end{array}$ & $\begin{array}{l}45 X / \\
46 X Y\end{array}$ & $\mathrm{AB}$ & $\begin{array}{l}\text { IN } \\
\text { G }\end{array}$ & $\begin{array}{l}6 . \\
5\end{array}$ & DT & Streak & MGD & $\begin{array}{l}\text { Right } \\
\text { gonadectomy } \\
\text { with left } \\
\text { orchidopexy. }\end{array}$ \\
\hline 18 & Female & $\begin{array}{l}\text { Primary } \\
\text { Ammenohrrea }\end{array}$ & $46 X Y$ & $\mathrm{AB}$ & $\begin{array}{l}\text { IN } \\
\text { G }\end{array}$ & $\begin{array}{l}1 . \\
5\end{array}$ & Testis & Testis & CAIS & $\begin{array}{l}\text { Bilateral } \\
\text { gonadectomy }\end{array}$ \\
\hline 18 & Female & $\begin{array}{l}\text { Primary } \\
\text { Ammenohrrea }\end{array}$ & $46 X Y$ & ING & $\begin{array}{l}\text { IN } \\
\text { G }\end{array}$ & 2 & Testis & Testis & CAIS & $\begin{array}{l}\text { Bilateral } \\
\text { gonadectomy }\end{array}$ \\
\hline 18 & Female & $\begin{array}{l}\text { Primary } \\
\text { Ammenohrrea }\end{array}$ & $46 X Y$ & ING & $\begin{array}{l}\text { IN } \\
\text { G }\end{array}$ & 2 & Testis & Testis & CAIS & $\begin{array}{l}\text { Bilateral } \\
\text { gonadectomy }\end{array}$ \\
\hline 20 & Female & $\begin{array}{l}\text { Primary } \\
\text { infertility }\end{array}$ & $46 X Y$ & ING & $\begin{array}{l}\text { IN } \\
\text { G }\end{array}$ & 2 & Testis & Testis & CAIS & $\begin{array}{l}\text { Bilateral } \\
\text { gonadectomy }\end{array}$ \\
\hline 20 & Female & $\begin{array}{l}\text { Primary } \\
\text { infertility }\end{array}$ & $46 X Y$ & ING & $\begin{array}{l}\text { IN } \\
\text { G }\end{array}$ & 1 & Testis & Testis & CAIS & $\begin{array}{l}\text { Bilateral } \\
\text { gonadectomy }\end{array}$ \\
\hline 20 & Female & $\begin{array}{l}\text { Primary } \\
\text { infertility }\end{array}$ & $46 X Y$ & ING & $\begin{array}{l}\text { IN } \\
\text { G }\end{array}$ & 2 & Testis & Testis & CAIS & $\begin{array}{l}\text { Bilateral } \\
\text { gonadectomy }\end{array}$ \\
\hline 22 & Female & $\begin{array}{l}\text { Primary } \\
\text { infertility }\end{array}$ & $46 X Y$ & $\mathrm{AB}$ & $\mathrm{AB}$ & 1 & Testis & Testis & CAIS & $\begin{array}{l}\text { Bilateral } \\
\text { gonadectomy }\end{array}$ \\
\hline 24 & Female & $\begin{array}{l}\text { Primary } \\
\text { infertility }\end{array}$ & $46 X Y$ & ING & $\mathrm{AB}$ & $\begin{array}{l}1 . \\
5\end{array}$ & Streak & Streak & CAIS & $\begin{array}{l}\text { Bilateral } \\
\text { gonadectomy }\end{array}$ \\
\hline 28 & Female & $\begin{array}{l}\text { Primary } \\
\text { infertility }\end{array}$ & $46 X Y$ & ING & $\begin{array}{l}\text { IN } \\
\text { G }\end{array}$ & 2 & Testis & Testis & CAIS & $\begin{array}{l}\text { Bilateral } \\
\text { gonadectomy }\end{array}$ \\
\hline 12 & Female & Primary & $46 X Y$ & $\mathrm{AB}$ & IN & 1. & DT & DT & PGD & Bilateral \\
\hline
\end{tabular}

Continued. 


\begin{tabular}{|c|c|c|c|c|c|c|c|c|c|c|}
\hline 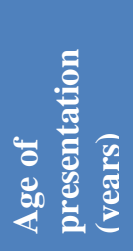 & 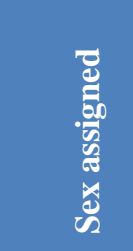 & 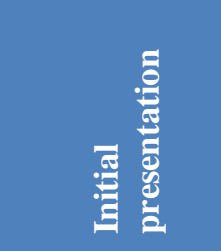 & 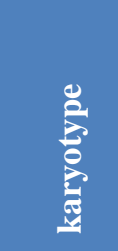 & 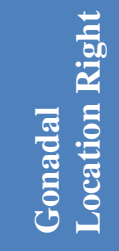 & 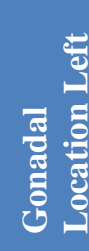 & $\sum_{i=1}^{\infty}$ & 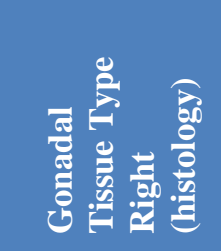 & 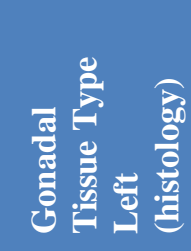 & 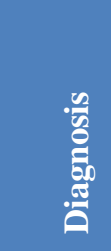 & 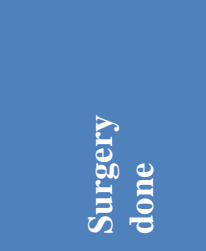 \\
\hline & & Ammenohrrea & & & G & 5 & & & & gonadectomy \\
\hline 16 & Female & $\begin{array}{l}\text { Primary } \\
\text { Ammenohrrea }\end{array}$ & $46 X Y$ & $\mathrm{AB}$ & $\begin{array}{l}\text { IN } \\
\text { G }\end{array}$ & $\begin{array}{l}1 . \\
5\end{array}$ & DT & DT & PGD & $\begin{array}{l}\text { Bilateral } \\
\text { gonadectomy }\end{array}$ \\
\hline 12 & Male & $\begin{array}{l}\text { Ambigious } \\
\text { genitals }\end{array}$ & $46 X Y$ & $\mathrm{~S}$ & $\mathrm{~S}$ & 9 & Testis & Testis & $5 \mathrm{AR}$ & $\begin{array}{l}\text { Chordee } \\
\text { correction }\end{array}$ \\
\hline 12 & Male & $\begin{array}{l}\text { Ambigious } \\
\text { genitals }\end{array}$ & $46 X Y$ & $\mathrm{~S}$ & $S$ & 9 & Testis & Testis & $5 \mathrm{AR}$ & $\begin{array}{l}\text { Hypospadias } \\
\text { correction }\end{array}$ \\
\hline 12 & Male & $\begin{array}{l}\text { Ambigious } \\
\text { genitals }\end{array}$ & $46 X Y$ & $\mathrm{~S}$ & $S$ & 10 & $\begin{array}{l}\text { Testis } \\
\text { Primary } \\
\text { Ammenoher } \\
\text { ea }\end{array}$ & Testis & $5 \mathrm{AR}$ & $\begin{array}{l}\text { Hypospadias } \\
\text { and chordee } \\
\text { correction }\end{array}$ \\
\hline 12 & Male & $\begin{array}{l}\text { Ambigious } \\
\text { genitals }\end{array}$ & $46 X Y$ & S & S & 9 & Testis & Testis & $5 \mathrm{AR}$ & $\begin{array}{l}\text { Hypospadias } \\
\text { and chordee } \\
\text { correction }\end{array}$ \\
\hline 12 & Male & $\begin{array}{l}\text { Ambigious } \\
\text { genitals }\end{array}$ & $46 X Y$ & S & $\mathrm{S}$ & 9 & Testis & Testis & $5 \mathrm{AR}$ & $\begin{array}{l}\text { Chordee } \\
\text { correction }\end{array}$ \\
\hline 12 & Male & $\begin{array}{l}\text { Ambigious } \\
\text { genitals }\end{array}$ & $46 X Y$ & S & $S$ & 8 & Testis & Testis & $5 \mathrm{AR}$ & $\begin{array}{l}\text { Hypospadias } \\
\text { and chordee } \\
\text { correction }\end{array}$ \\
\hline 16 & Male & $\begin{array}{l}\text { Ambigious } \\
\text { genitals }\end{array}$ & $46 X Y$ & $\mathrm{~S}$ & $S$ & 11 & Testis & Testis & $5 \mathrm{AR}$ & $\begin{array}{l}\text { Hypospadias } \\
\text { correction }\end{array}$ \\
\hline 16 & Male & $\begin{array}{l}\text { Ambigious } \\
\text { genitals }\end{array}$ & $46 X Y$ & $\mathrm{~S}$ & $S$ & 11 & Testis & Testis & $5 \mathrm{AR}$ & $\begin{array}{l}\text { Hypospadias } \\
\text { correction }\end{array}$ \\
\hline 14 & Male & Gynecomsatia & $46 X Y$ & S & $S$ & 8 & Testis & ING & $\begin{array}{l}17 \mathrm{BH} \\
\mathrm{SD}\end{array}$ & $\begin{array}{l}\text { Hypospadias } \\
\text { correction }\end{array}$ \\
\hline
\end{tabular}

Table 2: Psychosexual assessment $(n=32)$.

\begin{tabular}{|lllll|}
\hline Age of presentation (years) & Sex assigned at birth & Gender identity & Gender role & Sexual orientation \\
\hline 14 & Female & Female & Female & Heterosexual \\
\hline 14 & Female & Female & Female & Heterosexual \\
\hline 14 & Female & Female & Female & Heterosexual \\
\hline 14 & Female & Female & Female & Heterosexual \\
\hline 16 & Female & Female & Female & Heterosexual \\
\hline 16 & Female & Female & Female & Heterosexual \\
\hline 4 & Female & Female & Female & Heterosexual \\
\hline 8 & Female & Female & Female & Heterosexual \\
\hline 10 & Female & Female & Female & Heterosexual \\
\hline 12 & Female & Female & Female & Heterosexual \\
\hline 14 & Female & Female & Female & Heterosexual \\
\hline 16 & Male & Male & Male & Heterosexual \\
\hline 18 & Female & Female & Female & Heterosexual \\
\hline 18 & Female & Female & Female & Heterosexual \\
\hline 18 & Female & Female & Female & Heterosexual \\
\hline 20 & Female & Female & Female & Heterosexual \\
\hline 20 & Female & Female & Female & Heterosexual \\
\hline 20 & Female & Female & Female & Heterosexual \\
\hline 22 & Female & Female & Female & Heterosexual \\
\hline 24 & Female & Female & Female & Heterosexual \\
\hline
\end{tabular}

Continued. 


\begin{tabular}{|lllll|}
\hline Age of presentation (years) & Sex assigned at birth & Gender identity & Gender role & Sexual orientation \\
\hline 28 & Female & Female & Female & Heterosexual \\
\hline 12 & Female & Female & Female & Heterosexual \\
\hline 16 & Female & Female & Female & Heterosexual \\
\hline 12 & Male & Male & Male & Heterosexual \\
\hline 12 & Male & Male & Male & Heterosexual \\
\hline 12 & Male & Male & Male & Heterosexual \\
\hline 12 & Male & Male & Male & Heterosexual \\
\hline 12 & Male & Male & Male & Heterosexual \\
\hline 12 & Male & Male & Male & Heterosexual \\
\hline 16 & Male & Male & Male & Heterosexual \\
\hline 16 & Male & Male & Male & Heterosexual \\
\hline 14 & Male & Male & Male & Heterosexual \\
\hline
\end{tabular}

\section{DISCUSSION}

The development of the male sex is as a result of the interplay between products of SRY genes located on the $\mathrm{Y}$ chromosome. ${ }^{4}$ Bipotential gonad differentiates into testis followed by development of internal wolffian ducts and external male genitalia. Sertoli cells are the earliest testicular tissue to develop and it secrete anti-Mullerain hormone (AMH) around 6 to 7 weeks of gestation. ${ }^{5} \mathrm{AMH}$ cause regression of Mullerian ducts. Leydig cells from the fetal testis produce testosterone, which promotes the development and differentiation of the Wolffian ducts like epididymis, seminal vesicle and vas deference.

The external genitals and prostate development occur under the influence of 5- hydrotestosterone (5HT). Any insult at the testicular development, androgen production or its action leads to a broad range of clinical presentation called 46 XY DSD.

46 XY CGD are born with female phenotype. They have normal height to tall stature with no breast, pubic or axillary hair developed.

They usually present at adolescent as primary amenorrhea. They don't virilise at puberty due to the complete absence of testicular function. They have Mullerian structures due to absence of AMH. On the other hand subjects with 45,X/46,XY or MGD and PGD may have a spectrum of genital phenotype. The spectrum of genital phenotype depends on the degree of testicular function. Children with MGD present as ambiguous genitals at birth, a female child virilising before or at puberty, features of turner's stigmata or rarely as primary amenorrhea. ${ }^{6}$ The age of presentation may be wide unlike CGD who tend to present during puberty due to amenorrhea. In addition, subjects with MGD may present with Turners stigmata which include growth failure, cardiac and renal anomalies. ${ }^{7}$

Individuals with CGD, MGD and PGD are at high risk to develop gonadal tumors which arise from dysgenetic gonads. The risk of gonadal malignancy is $18 \%$ among MGD, $30 \%$ among CGD and $54 \%$ among PGD. ${ }^{8,9}$ This compels the need for early gonadectomy in this group of patients. Gender assignment depends on patient choice and based on the internal and external urogenital anatomy. In our series all cases of CGD, PGD and five cases of MGD were assigned and raised as females and they wished to continue so. All cases of CGD, PGD, CAIS and one five cases of MGD wanted to continue as females and underwent bilateral gonadectomy and feminising hormones were started in who have completed 12 years of age. One case of MGD (number 9) underwent clitoral recession (clitoral size $4 \mathrm{~cm}$ ) and other cases of MGD with clitoromegaly were monitored, and they had a spontaneous resolution of clitoral size after gonadectomy. One case of MGD (number 12) who was raised as a male and had a preference to female partner underwent unilateral right gonadectomy with left inguinal testis biopsy and orchidopexy. This case had suboptimal levels of total testosterone levels $(128 \mathrm{ng} / \mathrm{dl})$ and hence started on testosterone therapy.

All the CAIS subjects presented as the absence of menstruation despite normal developed breast and scanty pubic hair. This presentation has been the classic description. CAIS subjects had testis either located in the abdomen or in inguinal region. After understanding the gender preference subjects with CAIS underwent bilateral gonadectomy.

The timing of gonadectomy is a question yet to be solved. A study by Liu et al has shown the chances of gonadal tumors like gonadoblastoma is as high as $30 \%$ by the age 20 years. ${ }^{10}$ On the other hand Cools et al showed that there were no chances of development of testicular tumors or even carcinoma in-situ and the occurrences of pre- germ cell neoplasia in situ (pre-GCIS) lesion was only $14 \%$ by 16 years of age. ${ }^{11,12}$

Learning these data from studies on CAIS it would be better to carry out a biopsy of the testis if patients prefer gonadectomy in a later age. Ruling out malignancy by biopsy can help us to defer gonadectomy to later years. One subject presented with gynecomastia (Number 32) and had ambiguous genitals with an EMS of 8. This case was later found to be 17 BHSD deficiencies. 
Gynecomastia at puberty is a manifestation of the 17 BHSD deficiency and occurs due to conversion of androstenedione by aromatase in extraglandular tissue. ${ }^{13}$ This subject improved with tamoxifen therapy. Also subject with 17 BHSD3 deficiencies (number 32) had lower levels of total testosterone levels were also started on testosterone therapy after complete resolution of gynecomastia.

Lower total testosterone levels are attributed by deficiency of 17 BHSD3 enzyme deficiency which converts $\mathrm{A}$ to $\mathrm{T}$. 5alpha reductase 2 enzymes are expressed in prostate and in external genitalia. Its function is the conversion of testosterone to $5 \mathrm{HT}$. 5HT muscularize external genitals. Its deficiency leads to minor ambiguity of the genitals or hypospadia with or without blind vaginal pouch.

They have normal testis and wolffian ducts. Corrective surgeries for chordee, hypospadia and urethroplasty may be needed depending on the structural defects. We would also like to make a note of the limitation of this retrospective data. The limitation of the study was genetic analysis like androgen receptor mutational analysis and gene mutation for androgen biosynthetic defects were not available. Genetic analysis is essential at times for confirming diagnosis and for genetic counselling.

\section{CONCLUSION}

$46 \mathrm{XY}$ DSD's may share similarities in their clinical presentation but the age of presentation is wide. The definitive diagnosis needs radiological, laparoscopies and histological evaluation to ascertain the type of internal genital ducts and gonadal type. Management of these patients is multidisciplinary which needs an endocrinologist, gynaecologist, psychologist, pediatrician and clinical genetician. Proper gender assignment, gonadectomy, reconstructive surgeries, hormone replacement and time to time follow up for testicular malignancy in cases where testis are preserved is the ideal management. We may need a long term follow up of these subjects to study the fertility prospects in future.

Funding: No funding sources

Conflict of interest: None declared

Ethical approval: The study was approved by the Institutional Ethics Committee IRB Number: HEC.No.05/18/2020MCT Substudy

\section{REFERENCES}

1. Lee PA, Houk CP. Ahmed SF. Consensus statement on management of intersex disorders. International consensus conference on intersex. Pediatrics. 2006;118:e488-e500.
2. Goyal A, Kubihal S, Gupta Y. Dynamic testing for evaluation of adrenal and gonadal function in pediatric and adult endocrinology: An overview. Indian J EndocrMetab. 2019;23:593-601.

3. Ahmed SF, Khwaja O, Hughes IA. The role of a clinical score in the assessment of ambiguous genitalia. BJU Int. 2000;85:120-12.

4. Lovell-Badge R. The role of Sry in mammalian sex determination. Ciba Found Symp. 1992;165:162-79.

5. Rey R, Lukas-Croisier C, Lasala C, Bedecarrás $P$. AMH/MIS: What we know already about the gene, the protein and its regulation. Mol Cell Endocrinol 2003;211:21-31.

6. Cools M, Boter M, van Gurp R, Stoop H, Poddighem P. Impact of the Y-containing cell line on histological differentiation patterns in dysgenetic gonads. Clinical Endocrinol. 2007;67:184-192.

7. Farrugia MK, Sebire NJ, Achermann JC, Eisawi A, Duffy PG, Mushtaq I. Clinical and gonadal features and early surgical management of $45, \mathrm{X} / 46, \mathrm{XY}$ and 45,X/47,XYY chromosomal mosaicism presenting with genital anomalies. J Pediatr Urol. 2013;9(2):139-144.

8. McCann-Crosby B, Mansouri R, Dietrich JE, McCullough LB, Sutton VR, Austin EG, et al. State of the art review in gonadal dysgenesis: Challenges in diagnosis and management. Int $\mathbf{J}$ Pediatr Endocrinol. 2014;2014:4.

9. Cools M, Pleskacova J, Stoop H. On behalf of the Mosaicism Collaborative Group, Gonadal Pathology and Tumor Risk in Relation to Clinical Characteristics in Patients with 45,X/46,XY Mosaicism, The Journal of Clinical Endocrinology \& Metabolism. 2011;96:1171-80.

10. Liu AX, Shi HY, Cai ZJ. Increased risk of gonadal malignancy and prophylactic gonadectomy: a study of 102 phenotypic female patients with Y chromosome or Y-derived sequences. Human Reproduction. 2014;29:1413-9.

11. Das DV, Jabbar PK, Gomez R, Seena TP. Hemoptysis: A rare presentation of mixed gonadal dysgenesis. J Hum Reprod Sci. 2020;13:242-4.

12. Das S, Saikia UK, Saikia KK, Sarma D, Choudhury BK, Bhuyan AK, et al. "Spectrum of $46 \mathrm{XY}$ disorders of sex development": A hospital based cross sectional study. Indian $\mathbf{J}$ Endocr Metab. 2020;24:360-5.

13. Cools M, Wolffenbuttel KP, Hersmus R. Malignant testicular germ cell tumors in postpubertal individuals with androgen insensitivity: prevalence, pathology and relevance of single nucleotide polymorphism-based susceptibility profiling, Human Reproduction. 2017;32:2561-73.

Cite this article as: Das DV. Clinical profile of patients with $46 \mathrm{XY}$ disorders of sex development: a single centered experience. Int J Reprod Contracept Obstet Gynecol 2021;10:1967-72. 